\title{
Materiales pedagógicos de la naturaleza como factores favorecedores del aprendizaje para mejorar la Calidad Educativa
}

\section{Pedagogical materials of nature as factors favoring learning to improve Educational Quality}

\author{
María Eugenia García-Alcívar \\ megag1981@hotmail.com \\ Ministerio de Educación del Ecuador \\ Ecuador \\ https://orcid.org/0000-0002-4387-6761 \\ Ana Karen Mera-Rodríguez \\ ankamero89@hotmial.com \\ Ministerio de Educación del Ecuador \\ Ecuador \\ https://orcid.org/0000-0001-6140-1906 \\ Yesenia Magdalena García-Delgado \\ yeseniagarciadelgado@hotmail.com \\ Ministerio de Educación del Ecuador \\ Ecuador \\ https://orcid.org/0000-0001-9771-6205
Marian Liccette Rodríguez-Mendoza lisorodriguez@live.com
Ministerio de Educación del Ecuador
Ecuador \\ https://orcid.org/0000-0002-2477-1008
}

Recibido: 1 de noviembre de 2019\}

Aprobado: 5 de diciembre de 2019 


\title{
RESUMEN
}

El objetivo general de la investigación es analizar los materiales pedagógicos de la naturaleza como factores favorecedores del aprendizaje para mejorar la calidad educativa. Se empleó un tipo de investigación descriptiva - documental, con diseño bibliográfico. Se debe configurar una óptica diferente de evaluación, basándose en la observación, demostración de competencias, habilidades, entre otros factores que permiten comprobar que el estudiante ha adquirido un conocimiento en pertinencia a las metas educativas institucionales, siendo este contextualizado con la realidad social donde se desenvuelve. Se puede concluir que al aplicar recursos metodológicos o pedagógicos por medio de la naturaleza facilita el aprendizaje de los alumnos, logrando ser investigadores de su entorno de enseñanza, desarrollando sus destrezas y habilidades, siendo creativos y con deseos de aprender aún más, valorando y respetando al medio ambiente como ente primordial para toda su vida.

Descriptores: aprendizaje activa; enseñanza y formación; Gestión de la educación; innovación educacional.

\begin{abstract}
The general objective of the research is to analyze the pedagogical materials of nature as factors favoring learning to improve the educational quality. A type of descriptive documentary research was used, with bibliographic design. A different perspective of evaluation must be configured, based on observation, demonstration of competencies, skills, among other factors that allow to verify that the student has acquired a knowledge in relevance to the institutional educational goals, being this contextualized with the social reality where unwrap It can be concluded that by applying methodological or pedagogical resources through nature, it facilitates students' learning, becoming researchers in their teaching environment, developing their skills and abilities, being creative and willing to learn even more, valuing and respecting to the environment as a primary entity for his whole life.
\end{abstract}

Descriptors: activity learning, Teaching and training, Educational management, Educational innovations. 


\section{INTRODUCCIÓN}

Los análisis a las formas tradicionales de enseñar han conseguido mucha más fuerza ante los requerimientos de una formación que faculte confrontar la rápida obsolescencia de las sapiencias y la necesidad de asegurar un óptimo, efectivo, aprendizaje, los cuales aporten al avance de las potencialidades de cada persona. El aprendizaje cimentado en el uso de materiales del ambiente resulta ser una propuesta de aula fundamentada en la resolución de problemas, el cual dentro de las escuelas estimulan los procesos de metacognición debido que hay un mayor grado de relación entre lo que los estudiantes conocen o les resulta conocido, así como lo nuevo o desconocido, es decir, se estimula una conexión entre el conocimiento cotidiano y el científico.

Dentro del ámbito educativo toda clase de material puede ser un recurso que contribuya con el desarrollo de la enseñanza y el aprendizaje, más no todo material se concibe implementarse para este fin. No obstante, hay un sin número de utensilios que son empleados en educación infantil con propósitos educativos, al igual que existe un cuantioso catálogo de instrumentos que se usan con fines formativos, aunque el propósito para los que fueron creados no haya sido pedagógico. Los docentes gracias a su creatividad, experiencia e imaginación han ido adaptando dichos materiales de acuerdo a las necesidades, características, de sus estudiantes con el propósito de alcanzar las capacidades propuestas dentro del sistema educativo.

En las instituciones educativas los educandos se encuentran con diferentes tipos de problemas, exigencias y también de oportunidades que quizás no habían tenido la oportunidad de vivir hasta el momento en sus hogares o comunidad. Las interacciones con los docentes, compañeros de clase, directivos y trabajadores de la institución, el análisis de algún asunto de interés, la indagación de un tema en común, son factores que de una $u$ otra forma repercuten en menor o mayor grado en su aprendizaje. Factores como estos son esencialmente los que aprovecha la formación basada en la utilización de materiales pedagógicos de la naturaleza para incrementar el desarrollo de 
las destrezas, habilidades, características de un ser más humano y social.

Los materiales pedagógicos elaborados con recursos que proporciona el medio natural, facilita la ejercitación de los niños para estimular las habilidades cognitivas como, clasificar, discernir semejanzas, diferencias para solucionar problemas, simultáneamente sirve para que los profesores se logren relacionar con sus estudiantes de mejor forma, dando lugar a que la oportunidad para que el procedimiento de enseñanza-aprendizaje sea mucho más extenso.

El medio ambiente, la naturaleza y el entorno inmediato proporcionan un sinfín de oportunidades que pueden ser gozadas tanto por los alumnos como por los docentes. Todo aquel elemento didáctico que se pueda elaborar con recursos del ambiente aportará experiencias de las cuales se puedan valer para abordar las distintas materias de los diferentes grados de la educación básica, otorgando a cada uno el margen de complejidad requerido según las sapiencias que los alumnos ya tienen. Cabe destacar que es importante investigar al comienzo de cada tema los conocimientos previos de los niños para poder divisar las nociones que ya poseen, con esto el docente podrá concluir cuáles son los materiales necesarios que cooperarán en la generación y ampliación de sus conocimientos, haciendo del aprendizaje un proceso con mayor significancia y profundidad.

Elaborar herramientas con recursos del medio ambiente proporciona mejor grado de eficacia dentro del aula, además la implementación de dichos recursos están al alcance los alumnos. Las diversas tramas que hay como las culturales, sociales y geográficos del medio facultan una pluralidad de ideas para la fabricación de numerosos materiales, los mismos que deben ser de fácil uso, no peligrosos, funcionales, visualmente llamativos, favorables para trabajos grupales e individuales y acorde a la edad e intereses de los estudiantes.

Calzadilla (2004) define los materiales en educación como "todo el amplio campo de los objetos que se ponen a disposición del niño" (p.93), de esta forma, todos los 
componentes que rodean a los niños tienen como principal función ser un factor intermedio entre sus conocimientos y la realidad en la que se desenvuelve, en resumen es como un portal por el cual los estudiantes comenzarán a explorar, expresar y descubrir su entorno.

\section{DESARROLLO}

\section{¿Qué es la pedagogía?}

A lo largo de la historia muchas son las maneras y formas de enseñar, la educación busca siempre mejorar la calidad de enseñanza aprendizaje hacia los estudiantes a través de técnicas, estrategias, métodos y otras maneras innovadoras. Es aquí, que se incluye la pedagogía como una herramienta para lograr el desarrollo humano y la construcción de nuevos conocimientos. Cossio (2014) indica que la pedagogía es la ciencia que estudia la metodología y las técnicas que son implementadas en la enseñanza del estudiante, especialmente en el aprendizaje. Al aplicar nuevas tecnologías en el formato del aprendizaje es una manera innovadora que despierta el interés en los alumnos en querer aprender.

La Pedagogía en la educación se le conoce como un fenómeno complejo, donde muestra conocimientos de diferentes ciencias y disciplinas que brindan la comprensión de lo que es la formación intelectual del estudiante. Esto implica comprender los procesos sistemáticos de aprendizaje como también el desarrollo de capacidades y habilidades que facilitan la toma de decisiones, para mejorar la calidad educativa inculcando los principios, métodos, prácticas y técnicas en los procesos de enseñanzaaprendizaje con el fin de obtener un aprendizaje significativo (Miña et al., 2018).

Con la aplicación de las diferentes definiciones que se les ha dado al aprendizaje, se determina que los estudiantes aprenden a través de las experiencias tanto escolares como familiares siendo estas actividades un estimulante a la curiosidad y al deseo de descubrir nuevos conocimientos a partir de lo que conoce, desarrollando sus destrezas 
y habilidades (García \& Vegas, 2019).

El desempeño del docente se ha ido transformando por medio de diferentes cambios, siendo un líder de sí mismo. El pedagogo como especialista en pedagogía ayuda a mejorar las metodologías dentro del procesos educativos, con la finalidad de favorecer al desarrollo de las sociedades, por esta razón se necesitan docentes actualizados, capaces de generar respuestas transformadoras a las nuevas exigencias, comprometidos a participar de manera activa perfeccionando su profesionalismo y así escalar enormemente la calidad educativa (Caride et al., 2015).

Es necesario que el docente sea un profesional altamente calificado, despertando el interés de los alumnos en el aula de clases, generando motivaciones para alcanzar los objetivos y preparar profesionales para la sociedad.

\section{Materiales pedagógicos}

El material didáctico ayuda en el proceso de aprendizaje en los alumnos, gracias al contacto práctico y lúdico que activan el gozo por aprender, que estimulando el desarrollo memorístico, así como la motricidad tanto fina y gruesa, lo cognitivo, lo físico, así como otros aspectos que son necesarios en el proceso evolutivo del estudiante. Además, estos materiales facilitan que el aprendizaje sea práctico y significativo, dependiendo de la aplicación metodológica que el docente haga en el salón de clases (Vargas, 2017).

Así mismo los materiales pedagógicos son todos los recursos que se obtienen del medio natural o su entorno que fortalecen el aprendizaje de los estudiantes. Al utilizar estos métodos dentro del ambiente educativo permiten mejorar la obtención de conceptos, habilidades, actitudes y destrezas del aprendizaje (Manrique \& Gallego, 2013).

En efecto, el aprendizaje que se genera en los estudiantes, gracias a la implementación de los materiales pedagógicos, evalúa el éxito de la labor del docente. Para ello, 
Moreno (2015) afirma que estas herramientas facilitan los aprendizajes de los niños, niñas y adolescentes generando fortalecer los saberes con mayor eficacia y valores morales. Es decir, que el uso de materiales didácticos en los educadores juega un papel fundamental en la enseñanza de los alumnos para que aprendan significativamente.

Cabero et al. (2018) describen que los recursos materiales funcionan al emplearse dentro del currículo en el proceso de enseñanza/aprendizaje son múltiples. Cualquier recurso pueden convertirse en un material o herramienta educativa, garantizando el cumplimiento de una serie de características que respondan a unos determinados aprendizajes.

De tal manera, González (2015) establece tres tipos de características que ha de emplearse en todo material que se utilice para la educación:

Función de apoyo al aprendizaje, los materiales benefician los contenidos de manera eficaz y significativa dentro del proceso de incorporación de aprendizaje.

Función estructuradora, facilita el ordenamiento de la información que reciben, consiguiendo, que los alumnos adquieran aprendizajes más específicos y concretos.

Función motivadora, cualquier recurso que se utilice en la educación de los alumnos tiene que ser llamativo, que logre una interacción y que favorezca el aprendizaje de manera agradable, divertida y placentera, donde el estudiante considere esta actividad académica aporte para la vida diaria.

Son muchos los factores que hay que tener en cuenta a la hora de seleccionar el material pedagógico o didáctico que va a ayudar en la adquisición de las competencias educativas, lo primordial es que el recurso pedagógico sea atractivo, funcional y que permita la interacción activa del niño o niña durante la clase; es decir, que potencie y fortalezca el nivel cognitivo en todo su proceso de enseñanza y aprendizaje. 


\section{Calidad educativa}

Al hablar de calidad educativa nos referimos al perfeccionamiento de todo el proceso de aprendizaje con la aplicación de metodologías, estrategias y técnicas que fortifican las destrezas de cada estudiante. Sobre todo, es considerada en ámbito educativo como el estudio de las formaciones como la estrategia de avance por excelencia para las instituciones educativas y sus alumnos (Noris et al., 2015).

García et al. (2018) indica que la calidad educativa es alta, cuando la comunidad valora de manera positiva los resultados e instrumentos de la educación, que la institución inculca en los estudiantes. Así mismo, esto responde a las necesidades y expectativas de la formación permanente de su capital intelectual generando alumnos con principios y valores humanos que sirven como base para la vida diaria.

Sin embargo, la institución educativa de calidad es la que siembra el progreso o avance de sus estudiantes en el progreso de logros intelectuales, sociales, morales y éticos, basado en su aprendizaje adquirido mediante el conocimiento previo. Se puede señalar que el sistema escolar eficaz o eficiente es el que maximiza la capacidad de las unidades educativas para alcanzar resultados favorables.

\section{Recursos de la naturaleza como materiales pedagógicos.}

En el proceso de enseñanza aprendizaje es importante implementar nuevos elementos como recursos o materiales pedagógicos partiendo de la investigación y exploración del medio natural, donde los estudiantes encontraran las respuestas a todas sus preguntas, todo esto interactuando con el medio natural (Arce, 2015).

Otra opción dentro de este proceso está la elaboración de materiales de aprendizajes como el medio que permitan al educador y la educadora realizar las tareas de su labor docente dentro del aula con mayores niveles de eficiencia, ya que contribuyen a que los recursos estén al alcance de los estudiantes y, de esta forma, puedan desarrollar diferentes actividades y alcanzar sus aprendizajes. Los materiales influyen en este 
proceso de aprendizaje cuando son utilizados con frecuentemente; por eso es necesario que los alumnos puedan verlos, manipularlos y utilizarlo con la orientación dirección adecuada del docente, permitiendo que el estudiante adquiera experiencias, valores y actitudes (Vargas, 2009).

\section{Tipos de recursos}

La naturaleza provee de diversos materiales los cuales se pueden emplear como recursos pedagógicos dentro del proceso de enseñanza aprendizaje. Delgado (2015) señala los materiales pedagógicos que más uso se les puede dar se encuentran los siguientes:

Piedras, permiten el conteo en el desarrollo lógico matemático de los niños, determinar su forma y característica como investigación, es decir un análisis del entorno.

Algodón, favorece la educación artística, como desarrollo creativo en cada uno de los estudiantes más que todo en los primeros aprendizajes.

Paisaje, mediante la percepción se puede definir las cualidades que posee medio natural, este aporta mejor comprensión sobre los recursos ecológicos los cuales derivan cambios a través de la tecnología que son factores que unifican todo el esfuerzo de la comunidad científica

Semillas, con su uso permiten estimular en el niño el pensamiento, la lectura y escritura, por sus diferentes tamaños, formas, color, textura, resultan ser un potencial innovador de apoyo dentro del proceso enseñanza y aprendizaje, principalmente en la educación inicial y los primeros grados de educación básica. La ventaja de trabajar con este tipo de recuso es porque son fáciles de conseguir y no son tóxicos, la desventaja sería el tamaño de las semillas pueden resultar peligrosa para los niños pequeños, por lo que se recomienda la vigilancia del docente, sin dejar de usarlas, ya que con ella el niño puede discriminar tamaño, 
formas y colores, también puede seriar, clasificar, contar permitiendo el desarrollo de su sicomotricidad

Arenero, es un espacio de juego con arena, ubicado dentro de las escuelas y parques. Esta puede tener diferentes tamaños y formas. La arena es un novedoso material de juego que invita al niño a usar la imaginación y experimentar con el mundo que le rodea. Además, el desarrollo motriz de los estudiantes se logra con la manipulación de la arena como herramienta didáctica, creando el uso de la imaginación y experimentación con el entorno que le rodea. De tal manera que el estudiante puede jugar con la arena en el parque o en la playa, pero también pueden montar un arenero en casa. Manipular la arena estimula las destrezas como la curiosidad y la creatividad y fortalece la motricidad fina y gruesa reconociendo su textura cuando la arena está seca o húmeda (Herrera y Valencia, 2016).

Todos los materiales del medio natural son el principio de un aprendizaje significativo, por cuanto los niños y niñas aprenden por medio de la observación y manipulación directa con lo que se está tratando del tema de clases, logrando fortalecer el proceso de enseñanza (Gómez, 2013).

\section{MÉTODO}

Se empleó un tipo de investigación descriptiva - documental, con diseño bibliográfico, siguiendo lo planteado por Hernández, Fernández y Baptista (2008), teniéndose como población, una selección de investigaciones publicadas en revistas arbitradas, tesis, a quienes se les aplicó la técnica de análisis de contenido con la finalidad de lograr el objetivo general de la investigación de analizar los materiales pedagógicos de la naturaleza como factores favorecedores del aprendizaje para mejorar la calidad educativa. 


\section{RESULTADOS}

En esta investigación los autores determinan diferentes metodologías y recursos que se pueden conocer para el uso de materiales pedagógicos de la naturaleza como factores que favorecen el aprendizaje para mejorar la calidad educativa; despertando el interés de los estudiantes en aprender de la manera dinámica e innovadora durante el proceso de enseñanza - aprendizaje. En la tabla 1 se especifica el análisis de cada uno de los autores.

\section{Cuadro 1}

Análisis de los autores citados.

\begin{tabular}{|c|c|c|c|}
\hline Autor & Tema & Fecha & Interpretación \\
\hline $\begin{array}{l}\text { Cabero et } \\
\text { al. }\end{array}$ & $\begin{array}{l}\text { Material educativo } \\
\text { multimedia para el } \\
\text { aumento } \\
\text { estrategias } \\
\text { metacognitivas de } \\
\text { comprensión } \\
\text { lectora. }\end{array}$ & 2018 & $\begin{array}{l}\text { Consideran que cualquier recurso } \\
\text { puede convertirse en un material o } \\
\text { herramienta educativa, garantizando el } \\
\text { cumplimiento de una serie de } \\
\text { características que respondan a unos } \\
\text { determinados aprendizajes. }\end{array}$ \\
\hline Cossio & $\begin{array}{l}\text { Pedagogía y calidad } \\
\text { de la educación }\end{array}$ & 2014 & $\begin{array}{l}\text { Expone que la pedagogía es la ciencia } \\
\text { que estudia la metodología y las } \\
\text { técnicas para implementarlas en la } \\
\text { enseñanza del estudiante, } \\
\text { especialmente en el aprendizaje } \\
\text { infantil. Despertando el interés en los } \\
\text { alumnos en querer aprender, llegando } \\
\text { ser una herramienta fundamental para } \\
\text { la evolución de la educación. }\end{array}$ \\
\hline
\end{tabular}


García et Gestión escolar y al. calidad educativa

2018

$\begin{array}{ll}\text { El paisaje en la } \\ \text { formación } & \text { de } \\ \text { maestros, } & \text { un }\end{array}$

Delgado recurso educativo de alto interés para la educación primaria

El material didáctico Manrique y para la construcción Gallego de aprendizajes significativos

Noris et al.

\section{Material didáctico para la evaluación del aprendizaje en} el Programa
Proceso de
Enseñanza 2015
Determinan que la comunidad educativa es la encargada de diagnosticar el resultado de la educación cuando detalla el valor positivo del aprendizaje de su representado, es ahí que indica que el aprendizaje es de calidad.

Considera que la naturaleza provee de diversos materiales los cuales se pueden emplear como recursos 2015 pedagógicos dentro del proceso de enseñanza aprendizaje.

Detallan que todos los recursos que se obtienen del medio natural logran fortalecer la enseñanza de los alumnos, permitiendo mejorar la obtención de conceptos, habilidades, actitudes y destrezas del aprendizaje. Indican que la calidad educativa se la considerada como la ciencia que estudia las formaciones como la estrategia de avance por excelencia para las instituciones educativas y sus alumnos, es decir inculcado aparte de 
Aprendizaje

impartido en

Tecnología de la

Salud aprendizajes significativos, formación de seres humanos con valores éticos y morales.

Con el desarrollo de los diferentes análisis que cada autor especificó en su investigación, demuestra que el uso de materiales pedagógicos de la naturaleza como factores que favorecen el aprendizaje para mejorar la calidad educativa, son esenciales para el progreso de habilidades y destrezas de los estudiantes, motivando el interés de atención a la clase como forma dinámica y novedosa en cada paso de aprendizaje.

\section{DISCUSIÓN}

En función de los resultados analizados, Silva (2018), se hace necesario contar la propuesta de Silva, quien considera que al utilizarse materiales innovadores para la generación de aprendizajes, se debe configurar una óptica diferente de evaluación, basándose en la observación, demostración de competencias, habilidades, entre otros factores que permiten comprobar que el estudiante ha adquirido un conocimiento en pertinencia a las metas educativas institucionales, siendo este contextualizado con la realidad social donde se desenvuelve.

Por otro lado, lanni Gómez (2017), comenta que el docente para configurar un aprendizaje innovador, debe transcender el modelo mecanicista de enseñanza, para promover la investigación como centro del conocimiento, esto le permitirá constituir equipos de trabajos que promuevan desde una vertiente significativa, así se aprende para la vida, creando, innovando, constituyendo el rol de asesor, uno de los principales retos en asumir para promover una educación en pertinencia a las exigencias globales de cara a la educación 2030. 


\section{CONCLUSIÓN}

Alcanzar aprendizajes de calidad requiere de múltiples retos en cumplir con las exigencias que demanda la comunidad, en formar niños, adolescentes, jóvenes y adultos con buen desempeño para un futuro mejor. Se puede concluir que al aplicar recursos metodológicos o pedagógicos por medio de la naturaleza facilita el aprendizaje de los alumnos, logrando ser investigadores de su entorno de enseñanza, desarrollando sus destrezas y habilidades, siendo creativos y con deseos de aprender aún más, valorando y respetando al medio ambiente como ente primordial para toda su vida.

\section{REFERENCIAS CONSULTADAS}

1. Arce, G. (2015). El entorno natural como recurso didáctico para la enseñanza de ciencias naturales en el sexto año de educación general básica de la Unidad Educativa San Pablo de Guirainag, (tesis de grado). Universidad Politécnica Salesiana del Ecuador, Cuenca, Ecuador.

2. Cabero, J., Piñero, R., \& Reyes, M. (2018). Material educativo multimedia para el aumento de estrategias metacognitivas de comprensión lectora. Perfiles educativos, 40(159), 144-159. Recuperado de http://www.scielo.org.mx/scielo.php?script=sci_arttext\&pid=S0185$26982018000100144 \& \operatorname{lng}=e s \&$ tlng=es.

3. Calzadilla, R. (2004). La pedagogía como ciencia humanista: conocimiento de síntesis, complejidad y pluridisciplinariedad. Revista de Pedagogía, 25(72), 123148. Recuperado de http://ve.scielo.org/scielo.php?script=sci_arttext\&pid=S0798$97922004000100005 \& \operatorname{lng}=e s \&$ Ing $=e s$.

4. Caride, J., Gradaílle, R. \& Caballo, M. (2015). De la pedagogía social como educación, a la educación social como Pedagogía. Perfiles educativos, 37(148), 04-11. Recuperado de http://www.scielo.org.mx/scielo.php?script=sci_arttext\&pid=S0185$26982015000200016 \&$ Ing=es\&tlng=es.

5. Cossio, J. (2014). Pedagogía y calidad de la educación: una mirada a la formación del maestro rural. Sophia, 10(1), undefined-undefined. Recuperado de https://www.redalyc.org/articulo.oa?id=4137/413734078002 
6. Delgado, E. (2015). El paisaje en la formación de maestros, un recurso educativo de alto interés para la educación primaria. Universidad de Valladolid TABANQUE Revista pedagógica, 3(28), 117-138

7. García, F., Juárez, S. \& Salgado, L. (2018). Gestión escolar y calidad educativa. Revista Cubana de Educación Superior, 37(2), 206-216. Recuperado de http://scielo.sld.cu/scielo.php?script=sci_arttext\&pid=S0257$43142018000200016 \& \operatorname{lng}=e s \&$ tIng $=$ es.

8. García, M. y Vegas, H. (2019). Rincones pedagógicos: Nuevas estrategias para aprender y enseñar. Revista Interdisciplinaria de Humanidades, Educación, Ciencia y Tecnología, 5(1), 875 - 892.

9. Gómez, M. (2013). Las semillas un recurso didáctico para desarrollar la actitud y pensamiento científico desde la lectura y escritura en niños de 5 a 7 años de edad. UNT Instituto de Investigaciones Educacionales INDIE- ECOMUCHIKUNT,3 (1), 1-6

10.González, J. (2015). Criterios para el diseño de materiales multimedia educativos. Interamerican Journal of Psychology, 49(2), undefined-undefined. Recuperado de https://www.redalyc.org/articulo.oa?id=284/28446019002

11. Herrera, M. y Valencia, S. (2016). El arenero como recurso didáctico para desarrollar habilidades psicomotoras en los niños y niñas de inicial en la unidad educativa "Manuela Cañizares" de Santo Domingo en el año lectivo 2016. (Tesis de grado). Universidad Central del Ecuador sede Santo Domingo, Santo Domingo, Ecuador.

12. Hernández, R., Fernández, C., \& Baptista, P. (2008). Metodología de la investigación. México, Mc Graw Hill Hispanoamericana. Hill Internacional.

13. lanni Gómez., L. (2017). Miramda: Una propuesta educativa emergente desde la investigación. Revista Arbitrada Interdisciplinaria Koinonía, 2(3), 9-30. Recuperado https://fundacionkoinonia.com.ve/ojs/index.php/revistakoinonia/article/view/49

14. Manrique, A. y Gallego, A. (2013). El material didáctico para la construcción de aprendizajes significativos. Revista Colombiana de Ciencias Sociales, 4(1), 101108. 
15. Miña, L., O"Relly, D., García, C., Pérez, Z. \& Moreno, L. (2018). Papel de la Pedagogía del cambio en la Educación de Postgrado. Revista Médica Electrónica, 40(2), 445-453. Recuperado de http://scielo.sld.cu/scielo.php?script=sci_arttext\&pid=S1684$18242018000200019 \& \operatorname{lng}=\mathrm{es} \& \ln \mathrm{n}=\mathrm{es}$.

16. Moreno, F. (2015). Función pedagógica de los recursos materiales en educación infantil. Vivat Academia, (133), undefined-undefined. Recuperado de https://www.redalyc.org/articulo.oa?id=5257/525752885002

17. Noris, J., Vicente, J., Pérez, N., \& Noris, M. (2015). Material didáctico para la evaluación del aprendizaje en el Programa Proceso de Enseñanza - Aprendizaje impartido en Tecnología de la Salud. Correo Científico Médico, 19(4), 728-740. Recuperado en 25 de noviembre de 2019, de http://scielo.sld.cu/scielo.php?script=sci_arttext\&pid=S1560$43812015000400012 \& \operatorname{lng}=e s \&$ tIng=es.

18. Silva, C. (2018). Formación integral para facilitadores. EPISTEME KOINONIA, 1(1), 35-50. Recuperado de https://fundacionkoinonia.com.ve/ojs/index.php/epistemekoinonia/article/view/489 $\underline{1655}$

19. Vargas, C. (2009). Como elaborar un material didáctico. República Dominicana: INAFOCAM.

20. Vargas, G. (2017). Recursos educativos didácticos en el proceso enseñanza aprendizaje. Cuadernos Hospital de Clínicas, 58(1), 68-74. Recuperado de http://www.scielo.org.bo/scielo.php?script=sci_arttext\&pid=S1652-

$67762017000100011 \& \operatorname{lng}=$ es\&tlng=es.

\section{REFERENCES CONSULTED}

1. Arce, G. (2015). The natural environment as a teaching resource for the teaching of natural sciences in the sixth year of basic general education of the San Pablo de Guirainag Educational Unit (thesis). Salesian Polytechnic University of Ecuador, Cuenca, Ecuador.

2. Cabero, J., Piñero, R., \& Reyes, M. (2018). Multimedia educational material for the increase of metacognitive reading comprehension strategies. Educational profiles, $\quad 40 \quad$ (159), $144-159 . \quad$ Retrieved from 
http://www.scielo.org.mx/scielo.php?script=sci_arttext\&pid=S018526982018000100144\&lng=en\&tIng=en.

3. Calzadilla, R. (2004). Pedagogy as a humanistic science: knowledge of synthesis, complexity and multidisciplinarity. Pedagogy Magazine, 25 (72), 123148. Retrieved from http://ve.scielo.org/scielo.php?script=sci_arttext\&pid=S079897922004000100005\&lng=en\&tIng=en.

4. Caride, J., Gradaílle, R. \& Caballo, M. (2015). From social pedagogy as education, to social education as Pedagogy. Educational profiles, 37 (148), 04$11 . \quad$ Retrieved http://www.scielo.org.mx/scielo.php?script=sci_arttext\&pid=S0185$26982015000200016 \&$ Ing=en\&tIng=en.

5. Cossio, J. (2014). Pedagogy and quality of education: a look at the training of the rural teacher. Sophia, 10 (1), undefined-undefined. Recovered from https://www.redalyc.org/articulo.oa?id=4137/413734078002

6. Delgado, E. (2015). The landscape in teacher training, an educational resource of high interest for primary education. University of Valladolid TABANQUE Pedagogical Magazine, 3 (28), 117-138

7. García, F., Juárez, S. \& Salgado, L. (2018). School management and educational quality. Cuban Journal of Higher Education, 37 (2), 206-216. Retrieved from http://scielo.sld.cu/scielo.php?script=sci_arttext\&pid=S0257-

43142018000200016\&lng=en\&tlng=en.

8. García, M. and Vegas, H. (2019). Pedagogical corners: New strategies to learn and teach. Interdisciplinary Journal of Humanities, Education, Science and Technology, 5 (1), 875-892.

9. Gómez, M. (2013). Seeds a didactic resource to develop scientific attitude and thinking from reading and writing in children from 5 to 7 years old. UNT Educational Research Institute INDIE- ECOMUCHIK-UNT, 3 (1), 1-6

10. González, J. (2015). Criteria for the design of educational multimedia materials. Interamerican Journal of Psychology, 49 (2), undefined-undefined. Recovered from https://www.redalyc.org/articulo.oa?id=284/28446019002

11. Herrera, M. and Valencia, S. (2016). The sandbox as a didactic resource to develop psychomotor skills in initial children in the educational unit "Manuela 
Cañizares" of Santo Domingo in the 2016 school year. (Thesis). Central University of Ecuador, Santo Domingo headquarters, Santo Domingo, Ecuador.

12. Hernández, R., Fernández, C., \& Baptista, P. (2008). Investigation methodology. Mexico, Mc Graw Hill Hispanic American. Hill International

13. lanni Gómez., L. (2017). Miramda: An educational proposal emerging from research. Interdisciplinary Arbitrated Review Koinonía, 2 (3), 9-30. Recovered from

https://fundacionkoinonia.com.ve/ojs/index.php/revistakoinonia/article/view/49

14. Manrique, A. and Gallego, A. (2013). The teaching material for the construction of significant learning. Colombian Journal of Social Sciences, 4 (1), 101-108.

15. Miña, L., O "Relly, D., García, C., Pérez, Z. \& Moreno, L. (2018). Role of Pedagogy of Change in Postgraduate Education. Electronic Medical Journal, 40 (2), 445-453. Retrieved from http://scielo.sld.cu/scielo.php?script=sci_arttext\&pid=S1684$18242018000200019 \&$ Ing=en\&tlng=en.

16. Moreno, F. (2015). Pedagogical function of material resources in early childhood education. Vivat Academia, (133), undefined-undefined. Recovered from https://www.redalyc.org/articulo.oa?id=5257/525752885002

17. Noris, J., Vicente, J., Pérez, N., \& Noris, M. (2015). Teaching material for the evaluation of learning in the Teaching Process - Learning Program taught in Health Technology. Medical Scientific Mail, 19 (4), 728-740. Retrieved on November 25, 2019, from http://scielo.sld.cu/scielo.php?script=sci_arttext\&pid=S1560$43812015000400012 \& \operatorname{lng}=$ en\&tIng=en.

18. Silva, C. (2018). Integral training for facilitators. EPISTEME KOINONIA, 1 (1), 3550. https://fundacionkoinonia.com.ve/ojs/index.php/epistemekoinonia/article/view/489 1655

19. Vargas, C. (2009). How to develop a teaching material. Dominican Republic: INAFOCAM.

20.Vargas, G. (2017). Educational resources in the teaching-learning process. Cuadernos Hospital de Clínicas, 58 (1), 68-74. Retrieved from 


\section{CIENCIAMATRIA}

Revista Interdisciplinaria de Humanidades, Educación, Ciencia y Tecnología

Año VI. Vol. VI. N¹. Edición Especial. 2020

Hecho el depósito de ley: pp201602FA4721

ISSN-L: 2542-3029; ISSN: 2610-802X

Universidad Nacional Experimental Francisco de Miranda (UNEFM). Santa Ana de Coro. Venezuela

María Eugenia García-Alcívar; Ana Karen Mera-Rodríguez; Yesenia Magdalena García-Delgado;

Marian Liccette Rodríguez-Mendoza

http://www.scielo.org.bo/scielo.php?script=sci_arttext\&pid=S1652-

$67762017000100011 \& \operatorname{lng}=$ en\&tlng=en

(C2020 por los autores. Este artículo es de acceso abierto y distribuido según los términos y condiciones de la licencia Creative Commons Atribución-NoComercial-Compartirlgual 4.0 Internacional (CC BY-NC-SA 4.0) (https://creativecommons.org/licenses/by-nc-sa/4.0/). 